\title{
Effect of pulsed electromagnetic field therapy on experimental pain: a double-blind, randomized study in healthy young adults
}

\author{
Karen Beaulieu ${ }^{1}$, Patricia Beland ${ }^{1}$, Marilee Pinard ${ }^{1}$, Guilène Handfield ${ }^{2}$, Nicole \\ Handfield $^{2}$, Philippe Goffaux ${ }^{1,3}$; Helene Corriveau ${ }^{1,4}$, Guillaume Leonard ${ }^{1,4}$
}

${ }^{1}$ École de réadaptation de l'Université de Sherbrooke, Sherbrooke, Québec, Canada, J1H 5N4; ${ }^{2}$ Centre et services de conditionnement cellulaires, Beloeil, Québec, Canada, J3G 2V5; Centre de recherche du Centre hospitalier universitaire de Sherbrooke, Sherbrooke, Québec, Canada, J1H 5N4; ${ }^{4}$ Centre de recherche sur le vieillissement, Centre de santé et de services sociaux - Institut universitaire de gériatrie de Sherbrooke, Sherbrooke, Québec, Canada, J1H 4C4

Key words: pulsed electromagnetic field therapy, pain, experimental pain, temporal summation, thermode, physical therapy, rehabilitation

Corresponding author: Guillaume Léonard, pht, Ph.D.

Centre de recherche sur le vieillissement

Centre de santé et de services sociaux

Institut universitaire de gériatrie de Sherbrooke

1036, rue Belvédère Sud

Sherbrooke (Québec) J1H 4C4 


\begin{abstract}
Past studies have suggested that pulsed electromagnetic field (PEMF) therapy can decrease pain. To date, however, it remains difficult to determine if the analgesic effect observed in patients are attributable to a direct effect of PEMF on pain or to an indirect effect of PEMF on inflammation and healing. In the present study, we used an experimental pain paradigm to evaluate the direct effect of PEMF on pain intensity, pain unpleasantness and temporal summation of pain. Twenty-four healthy subjects (mean age $22 \pm 2$ years; 9 males) participated in the experiment. Both real and sham PEMF were administered to every participant using a randomized, double-blind, cross-over design. For each visit, PEMF was applied for 10 minutes on the right forearm using a portable device. Experimental pain was evoked before (baseline) and after PEMF with a $9 \mathrm{~cm}^{2}$ Pelletier type thermode, applied on the right forearm (120 sec stimulation; temperature individually adjusted to produce moderate baseline pain). Pain intensity and unpleasantness were evaluated using a 0-100 numerical pain rating scale. Temporal summation was evaluated by comparing pain intensity ratings obtained at the end of tonic nociceptive stimulation (120 sec) with pain intensity ratings obtained after $60 \mathrm{sec}$ of stimulation. When compared to baseline, there was no change in pain intensity and unpleasantness following the application of real or sham PEMF. PEMF did not affect temporal summation. The present observations suggest that PEMF does not directly influence heat pain perception in healthy individuals.
\end{abstract}




\section{Introduction}

Pulsed electromagnetic field (PEMF) therapy is a non-pharmacological modality that uses electromagnetic fields to accelerate tissue healing and foster recovery (Bachl et al. 2008; Markov 2007). In the past years, a number of studies have suggested that PEMF may also reduce pain (Bachl et al. 2008; Binder et al. 1984; Foley-Nolan et al. 1990; Kroeling et al. 2005). Although the physiological mechanisms responsible for PEMF analgesia remain uncertain, some authors have suggested that PEMF could influence pain via its effect on nitric oxide $(\mathrm{NO})$, calmodulin $(\mathrm{CaM})$ and/or opioid pathways (Abramson 2008; Diniz et al. 2002; Fitzsimmons et al. 2008; Pilla 2013; Staniland et al. 2010). To date however, results of studies looking into the effects of PEMF on pain have been inconsistent (Pieber et al. 2010; Ryang We et al. 2013; Teasell et al. 2010; Vavken et al. 2009). Given the effect of PEMF on tissue repair pathways (Callaghan et al. 2008;

Fitzsimmons et al. 2008; Pilla et al. 2011), it also remains difficult to determine if the analgesic effect observed in patients who received PEMF is attributable to a direct effect of PEMF on pain or to an indirect effect of PEMF on inflammation and healing.

Experimental pain paradigms are interesting research avenues that can be used to evaluate the analgesic effect of therapeutic interventions (Olesen et al. 2012). Many experimental pain paradigms allow for the assessment of pain and nociceptive responses in the absence of tissue injury or inflammation, thus offering a unique opportunity to evaluate the direct analgesic effect of a given intervention. To date, very few studies evaluated the analgesic effects of PEMF using experimental pain (Fernandez et al. 2007; Kortekaas et al. 2013; Shupak et al. 2004). 
Recently, Robertson et al. (2010) reported that the use of a whole-body electromagnetic field - applied during a painful stimulus with a strong static field of a magnetic resonance imaging instrument - decreases the activity of brain areas involved in the perception of the affective component of pain (Apkarian et al. 2005; Marchand 2010). Even though the results of Robertson et al. were obtained following whole-body electromagnetic field exposure rather than local electromagnetic field exposure (as is generally the case in clinical settings), their results raise the possibility that PEMF might preferentially influence the affective component of pain (pain unpleasantness), rather than the sensori-discriminative component of pain (pain intensity). In their study however, Robertson et al. did not report the effect of PEMF on subjective pain ratings, hence precluding any final conclusion regarding the effect of PEMF on the affective component of pain.

The lack of consensus regarding the analgesic effect of PEMF, as well as the absence of studies evaluating the effect of PEMF on the affective component of pain, emphasizes the necessity of conducting additional studies to assess the effect of PEMF on pain. In the present study, we evaluated the effect of PEMF on the two main components of pain (pain intensity and pain unpleasantness) by using an experimental tonic heat pain paradigm. The use of an experimental pain paradigm was preferred in order to exclude the effect that PEMF could have on inflammation and tissue repair. It also offered a unique opportunity to verify if PEMF could have an effect on temporal summation, an excitatory pain mechanism implicated in central sensitization and chronic pain (Katz and Rothenberg 2005; Vadivelu et al. 2010). 


\section{Methods}

\subsection{Participants}

Twenty-four healthy subjects (mean age $22 \pm 2$ years; 9 males) participated in this study. Participants were recruited through ads posted throughout Sherbrooke University's Health Campus. The experiment took place at the Clinique universitaire de réadaptation de l'Estrie, an outpatient clinic located in the Faculté de médecine et des sciences de la santé de l'Université de Sherbrooke (Canada). None of the participants suffered from any known diseases, had any recent musculoskeletal injury to the upper limbs, nor were they on any medication (except oral contraceptives). For security reasons, participants with a pacemaker or metal implants were excluded. Every participant was asked to refrain from consuming caffeine and smoking cigarettes $6 \mathrm{~h}$ before testing. The Research Ethics Board for the Research Center on Aging of the Health and Social Services Center - Sherbrooke University Geriatrics Institute, approved the study's procedures and each participant provided informed consent before participation.

\subsection{Tonic heat pain model}

Participants were seated comfortably in a reclining chair. Before each session, a pre-testing session was done in order to familiarize the participants with the thermode and to determine the temperature that would be used during the $2 \mathrm{~min}$ pain test. This pretest was performed with a $9 \mathrm{~cm}^{2}$ Peltier-type thermode (Medoc, Advanced Medical Systems, Ramat Yishai, Israel) applied on the ventral surface of the left forearm. Participants were told that the temperature of the thermode would gradually rise (from 32 
to $51^{\circ} \mathrm{C}$, rising rate $=0.3^{\circ} \mathrm{C} / \mathrm{sec}$ ). The pre-test procedure was repeated twice during which subjects verbally reported their pain perception and pain tolerance thresholds. On the third pre-test, subjects were given a computerized visual analogue scale (COVAS; Medoc, Advanced Medical Systems, Ramat Yishai, Israel) and advised that they would have to start moving the cursor towards to right (past the " 0 " mark) when they would start feeling pain (pain perception threshold) and that the cursor would need to be at the extreme right (at the "100" mark) when pain would become intolerable (pain tolerance threshold). This procedure was repeated until the subject's pain reports were consistent between trials. The temperature used during the following experimental heat pain test was determined by selecting the temperature for which the subject had rated the pain intensity at 50/100 with the COVAS (moderate pain).

Following the pre-test, participants were given a $10 \mathrm{~min}$ rest period before the experimental test began. The tonic pain test was then performed with the application of the thermode at constant temperature (determined during the pre-test) applied on the right forearm for $2 \mathrm{~min}$. Subjects were told that the temperature of the thermode could rise, remain stable or decrease and that they would have to verbally report pain intensity and unpleasantness with a 0-100 numerical rating scale (NRS) (pain intensity : $0=$ no pain, $100=$ unbearable pain; pain unpleasantness : $0=$ not at all unpleasant, $100=$ extremely unpleasant) every $15 \mathrm{~s}$ during the $2 \mathrm{~min}$ test. In fact, after a constant rise $\left(0.3^{\circ} \mathrm{C} / \mathrm{sec}\right)$ from baseline $\left(32^{\circ} \mathrm{C}\right)$ to the individually predetermined temperature, the thermode's temperature remained constant throughout the $120 \mathrm{sec}$. It was necessary to obtain pain measures every $15 \mathrm{sec}$ during the 2 min thermode test in order to evaluate temporal 
summation (Tousignant-Laflamme et al. 2008). The thermode test was repeated

immediately after the application of the PEMF, as well as every $15 \mathrm{~min}$ afterwards for the next hour. To avoid pain sensitization, the thermode was applied on an adjacent region of the right forearm, but never on the same site.

\subsection{PEMF application}

PEMF was administered on the right forearm, except in the case of one participant who received PEMF (and the thermode heat pain test) on the left forearm due to a past fracture of the right forearm. PEMF was delivered with a Rhumart Self-Health ${ }^{\mathrm{TM}}$ MBI3000 apparatus (SEM Electronique, Quebec, Canada) and two REBONE-4A applicators (Begue-Simon and Drolet 1993). Before the study, the Rhumart apparatus was evaluated by a certified operator to ensure proper calibration of the device. Both the real and sham interventions were applied for $10 \mathrm{~min}$. For the real intervention, the frequency was set at $60 \mathrm{~Hz}$ and the amplitude of the oscillating signal at 30\% of the maximal stimulator output $(30 \% \times 30$ Gauss $=9$ Gauss; continuous stimulation). The choice of the parameters was based on the manufacturer's recommendations and on the clinical expertise of the therapists ( $\mathrm{GH}$ and $\mathrm{NH})$. These parameters are comparable to the parameters used by Binder and colleagues (1984) who noted a favorable effect of PEMF on pain (over placebo) in a population of patients with persistent rotator cuff tendinitis. The same apparatus was used for the sham intervention, except that the applicators were connected to the second (inactive) channel. Thus, the lights of the apparatus were on, but no PEMF was delivered. Wooden chairs were used to avoid electromagnetic field interference. 


\subsection{Experimental design}

Participants were assigned in a double-blind, randomized, crossover design to receive, alternately, real and sham PEMF. Participants were enrolled in the study by KB, PB and MP, and were assigned to the treatment conditions by GL. The order of presentation of the two conditions was determined using a random number table, prepared by the statistician of the Research Center on Aging. The table was kept in all time in the office of the principal investigator (GL). This resulted in 12 participants receiving real PEMF before sham PEMF, and 12 participants receiving sham PEMF before real PEMF. Every participant was submitted to the two conditions (approximately 1-week interval). Both the participant and evaluator were blinded to the treatment being given. The tonic heat pain test was always performed without the person responsible for the application of PEMF being present.

\subsection{Statistical analysis}

The study was designed to detect a difference of 15 points on the NRS between real and sham PEMF. To detect this difference, with $80 \%$ power and a $5 \%$ significance level, we calculated that 24 participants had to be enrolled in the study (estimated standard deviation of 25 , based on preliminary work). Because of the relatively low number of subjects included in this study and because visual inspection of the histograms did not allow us to assume that the data were normally distributed, non-parametric tests were used. Specifically, Friedman's tests and Wilcoxon Signed-Rank tests were used to compare pain intensity and pain unpleasantness across the different time measures 
(before and after PEMF application) and between the two conditions for the same time measure (real and sham PEMF).

To compare temporal summation between the different time measures and determine if real PEMF affects excitatory pain mechanisms, we calculated delta scores (representing the difference between the pain intensity scores reported at $120 \mathrm{sec}$ and the pain intensity scores reported at $60 \mathrm{sec}$ of stimulation). Wilcoxon Signed-Rank tests were used to contrast the temporal summation values (delta scores) obtained before and after real PEMF. All tests were performed using SPSS (version 15.0 for Windows ${ }^{\circledR}$, Chicago, IL, USA). Differences were considered to be significant if $\mathrm{p}<0.05$ was obtained.

\section{Results}

\subsection{Baseline thermode measures}

Every participant were recruited and tested during the months of March and April 2012. All 24 participants completed the study and were included in the analyses. No important harms or side-effects were reported.

The pain intensity ratings obtained at baseline showed that every participant experienced pain during the thermode test (all pain intensity scores $>13$ ). The thermode temperatures used for the real and sham PEMF conditions were $46.2 \pm 1.5^{\circ} \mathrm{C}$ and $46.0 \pm$ $1.7^{\circ} \mathrm{C}$, respectively. A Wilcoxon Signed-Rank test revealed that there was no difference between these two temperatures $(\mathrm{p}=0.29)$.

\subsection{Effect of PEMF on pain intensity and pain unpleasantness}


The average pain intensity and pain unpleasantness scores obtained at baseline and at each time measure are presented in Figures 1A and 1B. As it can be seen from these figures, there was no change in pain intensity and pain unpleasantness after real or sham PEMF when compared to baseline (all p-values $>$.15). Head-to-head comparisons for each time measure revealed no difference between real and sham conditions (all p-values $>.10)$.

\subsection{Effect of PEMF on temporal summation}

The temporal summation observed at baseline and immediately after real PEMF application is shown in Figure 2. As it can be seen in this figure, there was a slight increase in pain during the last minute of stimulation for both the baseline and postPEMF conditions. Similar results were obtained for all time measures (i.e., at baseline, 0 min, $15 \mathrm{~min}, 30 \mathrm{~min}, 45 \mathrm{~min}$ and $60 \mathrm{~min}$ post-PEMF; see Figure 3), suggesting that PEMF had no effect on temporal summation. The Friedman test confirmed that there was no difference between the temporal summation delta scores (pain intensity at 120 seconds - pain intensity at 60 seconds) obtained at baseline, $0 \mathrm{~min}, 15 \mathrm{~min}, 30 \mathrm{~min}, 45$ $\min$ and 60 min post-PEFM $(\mathrm{p}=.74)$.

\subsection{Conditioning effect and influence of sex on PEMF analgesia Because previous} studies have observed that the conditioning effects provided by the experience of placebos can influence the results of clinical trials (Andre-Obadia et al. 2011; Leonard et al. 2010; Leonard et al. 2012), we performed between-subject analyses to determine if the pattern of analgesic response observed following real PEMF application was influenced 
by the order of presentation. To do this, delta pain scores, representing pain reductions experienced after real PEMF application (delta pain score $=$ pain at baseline - pain after PEMF), were calculated and compared between participants who received sham PEMF during their first session and those who received real PEMF during their first session. The analyses revealed that the order of presentation did not influence the pattern of results (i.e., similar analgesia following real PEMF for participants who received sham PEMF during their first visit and participants who received real PEMF during their first visit; $p>$ $.66)$.

The delta pain scores were also used to assess whether the sex of the participants influenced PEMF analgesia. The analysis showed that there was no difference in the delta pain scores between men and women (delta scores $=4 \pm 18$ and $6 \pm 20$, respectively; $p=$ 0.72), suggesting that sex had no effect on PEMF analgesia.

\section{Discussion}

In the present study, we examined whether experimental pain (induced by a tonic heat pain paradigm) could be modulated by PEMF. We observed no change in pain intensity, pain unpleasantness and temporal summation following PEMF application, suggesting that PEMF does not affect tonic experimental pain.

In the last decades, many studies have been conducted to evaluate the analgesic effect of PEMF. Positive results have been reported in various pain disorders, including inflammatory and post-operative pain conditions (Binder et al. 1984; Cheing et al. 2005;

Foley-Nolan et al. 1992; Heden and Pilla 2008; Uzunca et al. 2007). More contradictory 
findings have been observed for painful diabetic polyneuropathy, with some authors showing positive (Graak et al. 2009; Musaev et al. 2003) and others showing negative (Weintraub et al. 2009; Wrobel et al. 2008) results. Contradictory findings have also been reported for chronic pain conditions. For instance, Shupak and colleagues observed a beneficial effect of PEMF on pain in rheumatoid arthritis and fibromyalgia patients (Shupak et al. 2006), and similar results were reported by Foley and colleagues in patients suffering from persistent neck pain (Foley-Nolan et al. 1990). Alternately, others found no benefit for patients suffering of knee osteoarthritis (Ay and Evcik 2009; Ozguclu et al. 2010; Vavken et al. 2009) and chronic localized musculoskeletal pain (Thomas et al. 2007). These results suggest that PEMF may be useful to decrease pain in some, but not all, pain patient populations.

Interestingly, many conditions in which PEMF was shown to be effective (e.g., tendinitis, rheumatoid arthritis) are characterised by inflammatory pain (Binder et al. 1984; Cheing et al. 2005; Foley-Nolan et al. 1992; Heden and Pilla 2008; Uzunca et al. 2007). These observations raise the possibility that the analgesic effect of PEMF could be attributable to an effect of PEMF on inflammation. This hypothesis is supported by the results of Vincenzi and colleagues and Selvam and colleagues who observed that PEMF increases the anti-inflammatory effect of $\mathrm{A}_{2} \mathrm{~A}$ and $\mathrm{A}_{3}$ adenosine receptors (Petersen et al. 1988) and restores plasma membrane calcium ATPase activity (Grady et al. 1995). Future studies, studying the effect of PEMF on both pain and inflammatory markers are necessary before we can come to any final conclusions. 
Other studies have suggested that PEMF analgesia could be due to the effect of PEMF on NO and on the CaM signaling pathways (Diniz et al. 2002; Fitzsimmons et al. 2008; Pilla 2013). For instance, Fitzsimmons et al. (2008) showed that PEMF could promote the proliferation of chondrocytes via a CaM-dependant NO signaling cascade. Although these results suggest that the beneficial effect of PEMF on pain could be due to the effect of PEMF on chondrocytes and tissue repair, they do not exclude the possibility that PEMF could also directly influence pain. For example, in osteoarthritis patients, NO could improve blood flow to the affected joint (thus reducing ischemic pain) and activate opioid pathways (Abramson 2008; Staniland et al. 2010). The absence of analgesic effect noted in the present study suggests that the effect of PEMF on osteoarthritis pain may be due more to an effect of PEMF on chondrocytes and/or blood flow than to an effect on opioid pathways.

\subsection{Effect of PEMF on experimental pain}

Contrary to clinical pain paradigms, experimental pain paradigms allow researchers to standardize pain across participants and minimize time-related fluctuations in pain ratings. Many experimental pain paradigms also offer the advantage of avoiding tissue damage and inflammation, hence allowing researchers to determine if a given treatment directly influences pain perception. To our knowledge, very few studies looked at the effect of PEMF on experimental pain (Fernandez et al. 2007; Kortekaas et al. 2013; Shupak et al. 2004). In one study, conducted by Shupak et al. (2004), the authors evaluated the effect of PEMF on thermal non-nociceptive and nociceptive thresholds in a group of healthy volunteers. They observed that PEMF did not affect the thermal sensory 
threshold, but significantly increased thermal pain thresholds, hence suggesting a specific effect of PEMF on pain perception. These beneficial effects of PEMF on experimental pain were challenged by another study, conducted by Fernandez et al. (2007), who noted no difference in pain intensity between real and sham PEMF in a group of healthy volunteers who received hypertonic saline injections into the brachioradialis muscle of the non-dominant arm. In their study, Shupak and colleagues used pain thresholds to document the effect of PEMF on pain, while Fernandez and colleagues used a tonic pain stimulation paradigm (hypertonic saline infusion; perceived pain measured with a visual analog scale). These observations, as well as the results from the present study, indicate that the nature of the experimental paradigm (i.e., pain threshold $v s$ tonic pain procedure) can affect the pattern of results of PEMF pain studies. We believe that tonic pain paradigms more closely mimic clinical pain conditions than do research paradigms based on the evaluation of pain thresholds (Rainville et al. 1992).

\subsection{Pain unpleasantness}

In 2010, Robertson and colleagues (2010) evaluated the effect of a whole-body electromagnetic field on the activity of the cerebral structures implicated in pain perception. They noted that the increased cerebral activity observed during the application of an acute thermal pain paradigm was significantly reduced in participants who were exposed to a real electromagnetic field, compared to participants who were exposed to a sham electromagnetic field. These changes were noted in the insula and anterior cingulate cortex, two brain regions known to play a key role in the affective component of pain (Marchand 2010; Rainville et al. 1997). However, in their study, 
Robertson and colleagues did not measure pain intensity and pain unpleasantness, hence precluding any final conclusion regarding the effect that electromagnetic fields can have on subjective pain. In the present study, we observed no difference between real and sham PEMF for both pain intensity and pain unpleasantness. Our results suggest that PEMF does not affect the sensory-discriminative or motive-affective components of pain, at least when applied locally. Perhaps a more general application (i.e., whole-body field exposition), like the one used by Robertson and colleagues, could lead to different outcomes.

\subsection{Study limitations}

In this study, PEMF therapy was applied for 10 minutes with a frequency of $60 \mathrm{~Hz}$ and a field density of 9 Gauss. The choice of these parameters was based on the manufacturer's recommendations, the clinical expertise of the therapists ( $\mathrm{GH}$ and $\mathrm{NH}$ ) and the methodology of a previous PEMF study showing beneficial results (Binder et al. 1984). However, to date, the optimal stimulation parameters for PEMF have not yet been established. Different results would have perhaps been obtained if other PEMF parameters had been used.

Another important criticism that could be addressed to the present study is the use of an experimental pain paradigm rather than a clinical pain paradigm. Indeed, several of the previous studies looking at the effect of PEMF on pain employed clinical pain paradigms, and some could wonder if the results of this study (based on pain ratings obtained on healthy volunteers during prolonged heat stimulation) would also have been obtained in 
pain patients. Without refuting the fact that experimental pain paradigms have less external validity than clinical pain paradigms, one has to remember that the former has on the counterpart the advantage of increasing the internal validity due to the constant stimulation parameters employed at each time measure. As mentioned previously, the use of an experimental pain paradigm was preferred to a clinical pain paradigm in order to exclude the effect that PEMF could have on inflammation and tissue repair and better circumscribe the "true" effect of PEMF on pain. That being said, we must remember that the current results can only be applied to the pain modality used in the present study (i.e., tonic heat pain). Perhaps different results would be obtained with another pain model. Results from studies on transcutaneous electrical nerve stimulation (TENS), showing that the analgesic effect of TENS tends to vary across different pain models (see for instance Claydon et al. 2011 for a review) somewhat support such an interpretation. Future studies looking into the analgesic effect of PEMF with other experimental pain paradigms, should be conducted before any final conclusions can be drawn.

\section{Conclusion}

In conclusion, local application of PEMF does not appear to influence pain intensity and pain unpleasantness. Although it is still possible that PEMF may decrease pain in certain clinical conditions, the current results suggest that the analgesic effect noted with these patients is probably due to an effect of PEMF on inflammation and/or tissue healing, rather than to a direct effect of PEMF on pain. Future research is essential to confirm these results and to determine the optimal parameters of PEMF. 


\section{Declaration of interest}

PG, HC and GL are supported by FRQ-S (Quebec). GL and PG are supported by NSERC (Canada). The authors have no conflict of interest to report.

\section{Acknowledgements}

The authors wish to thank Alex Belletete and Amelyse Couture for their help with data collection. 


\section{References}

Abramson, S. B. (2008). Nitric oxide in inflammation and pain associated with osteoarthritis. Arthritis Res Ther 10:S2.

Andre-Obadia, N., Magnin, M., and Garcia-Larrea, L. (2011). On the importance of placebo timing in rTMS studies for pain relief. Pain 152:1233-1237.

Apkarian, A. V., Bushnell, M. C., Treede, R. D., and Zubieta, J. K. (2005). Human brain mechanisms of pain perception and regulation in health and disease. Eur J Pain 9:463484.

Ay, S., and Evcik, D. (2009). The effects of pulsed electromagnetic fields in the treatment of knee osteoarthritis: a randomized, placebo-controlled trial. Rheumatol Int 29:663-666.

Bachl, N., Ruoff, G., Wessner, B., and Tschan, H. (2008). Electromagnetic interventions in musculoskeletal disorders. Clin Sports Med 27:87-105.

Begue-Simon, A. M., and Drolet, R. A. (1993). Clinical assessment of the RHUMART system based on the use of Pulsed Electromagnetic Fields with low frequency. Int $\mathrm{J}$ Rehabil Res 16:323-327.

Binder, A., Parr, G., Hazleman, B., and Fitton-Jackson, S. (1984). Pulsed electromagnetic field therapy of persistent rotator cuff tendinitis. A double-blind controlled assessment. Lancet 1:695-698.

Callaghan, M. J., Chang, E.I., Seiser, N., Aarabi, S., Ghali, S., Kinnucan, E. R., Simon, B. J., and Gurtner, G. C. (2008). Pulsed electromagnetic fields accelerate normal and diabetic wound healing by increasing endogenous FGF-2 release. Plast Reconstr Surg 121:130-141.

Cheing, G. L., Wan, J. W., and Kai Lo, S. (2005). Ice and pulsed electromagnetic field to reduce pain and swelling after distal radius fractures. J Rehabil Med 37:372-377.

Claydon, L. S., Chesterton L. S., Barlas P., Sim J. (2011). Dose-specific effects of transcutaneous electrical nerve stimulation (TENS) on experimental pain: a systematic review. Clin J Pain 27:635-647.

Diniz, P., Soejima, K., and Ito, G. (2002). Nitric oxide mediates the effects of pulsed electromagnetic field stimulation on the osteoblast proliferation and differentiation. Nitric Oxide 7:18-23.

Fernandez, M. I., Watson, P. J., and Rowbotham, D. J. (2007). Effect of pulsed magnetic field therapy on pain reported by human volunteers in a laboratory model of acute pain. Br J Anaesth 99:266-269. 
Fitzsimmons, R. J., Gordon, S. L., Kronberg, J., Ganey, T., and Pilla, A. A. (2008). A pulsing electric field (PEF) increases human chondrocyte proliferation through a transduction pathway involving nitric oxide signaling. J Orthop Res 26:854-859.

Foley-Nolan, D., Barry, C., Coughlan, R. J., O'Connor, P., and Roden, D. (1990). Pulsed high frequency $(27 \mathrm{MHz})$ electromagnetic therapy for persistent neck pain. A double blind, placebo-controlled study of 20 patients. Orthopedics 13:445-451.

Foley-Nolan, D., Moore, K., Codd, M., Barry, C., O'Connor, P., and Coughlan, R. J. (1992). Low energy high frequency pulsed electromagnetic therapy for acute whiplash injuries. A double blind randomized controlled study. Scand J Rehabil Med 24:51-59.

Graak, V., Chaudhary, S., Bal, B. S., and Sandhu, J. S. (2009). Evaluation of the efficacy of pulsed electromagnetic field in the management of patients with diabetic polyneuropathy. Int J Diabetes Dev Ctries 29:56-61.

Grady, C. L., McIntosh, A. R., Horwitz, B., Maisog, J. M., Ungerleider, L. G., Mentis, M. J., Pietrini, P., Schapiro, M. B., and Haxby, J. V. (1995). Age-related reductions in human recognition memory due to impaired encoding. Science 269:218-221.

Heden, P., and Pilla, A. A. (2008). Effects of pulsed electromagnetic fields on postoperative pain: a double-blind randomized pilot study in breast augmentation patients. Aesthetic Plast Surg 32:660-666.

Katz, W. A., and Rothenberg, R. (2005). Section 3: The nature of pain: pathophysiology. J Clin Rheumatol 11:S11-5.

Kortekaas, R., van Nierop, L. E., Baas, V. G., Konopka, K. H., Harbers, M., van der Hoeven, J. H., van Wijhe, M., Aleman, A., and Maurits, N. M. (2013). A novel magnetic stimulator increases experimental pain tolerance in healthy volunteers - a double-blind sham-controlled crossover study. PLoS One 8:e61926.

Kroeling, P., Gross, A. R., Goldsmith, C. H., and Cervical Overview Group. (2005). A Cochrane review of electrotherapy for mechanical neck disorders. Spine (Phila Pa. 1976) 30:E641-8.

Leonard, G., Goffaux, P., and Marchand, S. (2010). Deciphering the role of endogenous opioids in high-frequency TENS using low and high doses of naloxone. Pain 151:215219.

Leonard, G., Lafrenaye, S., and Goffaux, P. (2012). Randomized placebo-controlled cross-over designs in clinical trials: a gold standard to be reassessed. Curr Med Res Opin 28:245-248.

Marchand, S. (2010). Applied pain neurophysiology. In Beaulieu, P., Lussier, D., Porreca, F. and Dickenson, A. H. eds. Pharmacology of Pain (pp. 3-26). Seattle: IASP Press. 
Markov, M. S. (2007). Expanding use of pulsed electromagnetic field therapies. Electromagn. Biol Med 26:257-274.

Musaev, A. V., Guseinova, S. G., and Imamverdieva, S. S. (2003). The use of pulsed electromagnetic fields with complex modulation in the treatment of patients with diabetic polyneuropathy. Neurosci Behav Physiol 33:745-752.

Olesen, A. E., Andresen, T., Staahl, C., and Drewes, A. M. (2012). Human experimental pain models for assessing the therapeutic efficacy of analgesic drugs. Pharmacol Rev 64:722-779.

Ozguclu, E., Cetin, A., Cetin, M., and Calp, E. (2010). Additional effect of pulsed electromagnetic field therapy on knee osteoarthritis treatment: a randomized, placebocontrolled study. Clin Rheumatol 29:927-931.

Petersen, S. E., Fox, P. T., Posner, M. I., Mintun, M., and Raichle, M. E. (1988). Positron emission tomographic studies of the cortical anatomy of single-word processing. Nature 331:585-589.

Pieber, K., Herceg, M., and Paternostro-Sluga, T. (2010). Electrotherapy for the treatment of painful diabetic peripheral neuropathy: a review. J Rehabil Med 42:289-295.

Pilla, A., Fitzsimmons, R., Muehsam, D., Wu, J., Rohde, C., and Casper, D. (2011).

Electromagnetic fields as first messenger in biological signaling: Application to calmodulin-dependent signaling in tissue repair. Biochim Biophys Acta 1810:1236-1245.

Pilla, A. A. (2013). Nonthermal electromagnetic fields: from first messenger to therapeutic applications. Electromagn. Biol Med 32:123-136.

Rainville, P., Duncan, G. H., Price, D. D., Carrier, B., and Bushnell, M. C. (1997). Pain affect encoded in human anterior cingulate but not somatosensory cortex. Science 277:968-971.

Rainville, P., Feine, J.S., Bushnell, M.C., and Duncan, G.H. (1992). A psychophysical comparison of sensory and affective responses to four modalities of experimental pain. Somatosens Mot Res 9:265-277.

Robertson, J. A., Theberge, J., Weller, J., Drost, D. J., Prato, F. S., and Thomas, A. W. (2010). Low-frequency pulsed electromagnetic field exposure can alter neuroprocessing in humans. J R Soc Interface 7:467-473.

Ryang We, S., Koog, Y. H., Jeong, K. I., and Wi, H. (2013). Effects of pulsed electromagnetic field on knee osteoarthritis: a systematic review. Rheumatology (Oxford) 52:815-824.

Shupak, N. M., McKay, J. C., Nielson, W. R., Rollman, G. B., Prato, F. S., and Thomas, A. W. (2006). Exposure to a specific pulsed low-frequency magnetic field: a double-blind 
placebo-controlled study of effects on pain ratings in rheumatoid arthritis and fibromyalgia patients. Pain Res Manag 11:85-90.

Shupak, N. M., Prato, F. S., and Thomas, A. W. (2004). Human exposure to a specific pulsed magnetic field: effects on thermal sensory and pain thresholds. Neurosci Lett 363:157-162.

Staniland, A. A., Walczak, J. S., and McMahon, S. B. (2010). Neurotrophic factors, neuropeptides and nitric oxide : therapeutic targets in chronic pain mechanisms. In Beaulieu, P., Lussier, D., Porreca, F. and Dickenson, A. H. eds. Pharmacology of Pain (pp. 253-278). Seattle: IASP Press.

Teasell, R. W., McClure, J. A., Walton, D., Pretty, J., Salter, K., Meyer, M., Sequeira, K., and Death, B. (2010). A research synthesis of therapeutic interventions for whiplashassociated disorder (WAD): part 2 - interventions for acute WAD. Pain Res Manag 15:295-304.

Thomas, A. W., Graham, K., Prato, F. S., McKay, J., Forster, P. M., Moulin, D. E., and Chari, S. (2007). A randomized, double-blind, placebo-controlled clinical trial using a low-frequency magnetic field in the treatment of musculoskeletal chronic pain. Pain Res Manag $12: 249-258$.

Tousignant-Laflamme, Y., Page, S., Goffaux, P., and Marchand, S. (2008). An experimental model to measure excitatory and inhibitory pain mechanisms in humans. Brain Res 1230:73-79.

Uzunca, K., Birtane, M., and Tastekin, N. (2007). Effectiveness of pulsed electromagnetic field therapy in lateral epicondylitis. Clin Rheumatol 26:69-74.

Vadivelu, N., Mitra, S., and Narayan, D. (2010). Recent advances in postoperative pain management. Yale J Biol Med 83:11-25.

Vavken, P., Arrich, F., Schuhfried, O., and Dorotka, R. (2009). Effectiveness of pulsed electromagnetic field therapy in the management of osteoarthritis of the knee: a metaanalysis of randomized controlled trials. J Rehabil Med 41:406-411.

Weintraub, M. I., Herrmann, D. N., Smith, A. G., Backonja, M. M., and Cole, S. P. (2009). Pulsed electromagnetic fields to reduce diabetic neuropathic pain and stimulate neuronal repair: a randomized controlled trial. Arch Phys Med Rehabil 90:1102-1109.

Wrobel, M. P., Szymborska-Kajanek, A., Wystrychowski, G., Biniszkiewicz, T., SieronStoltny, K., Sieron, A., Pierzchala, K., Grzeszczak, W., and Strojek, K. (2008). Impact of low frequency pulsed magnetic fields on pain intensity, quality of life and sleep disturbances in patients with painful diabetic polyneuropathy. Diabetes Metab 34:349354. 


\section{Figure Legends}

\section{Figure 1. Pain intensity and unpleasantness}

Pain intensity (A) and unpleasantness (B) were evaluated with a numerical pain rating scale $(0-100 \mathrm{~mm})$ before (baseline) and after PEMF application. When compared to baseline, there was no change in pain ratings after both real and sham PEMF (all p-values $>0.15)$

A

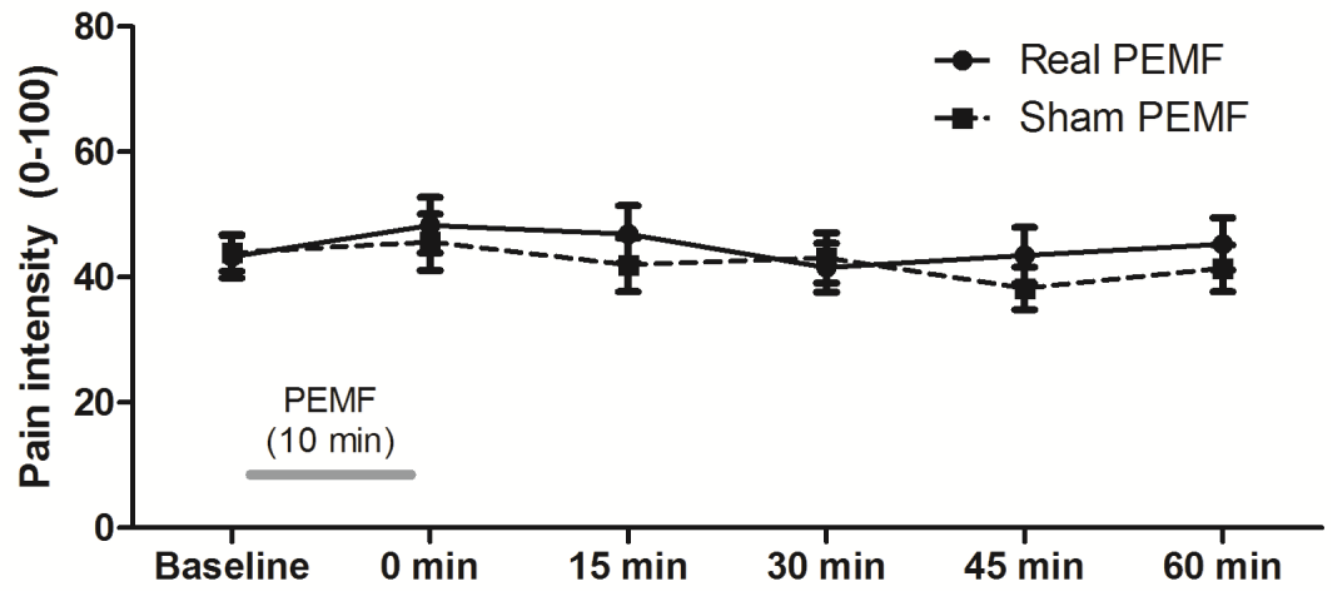

B

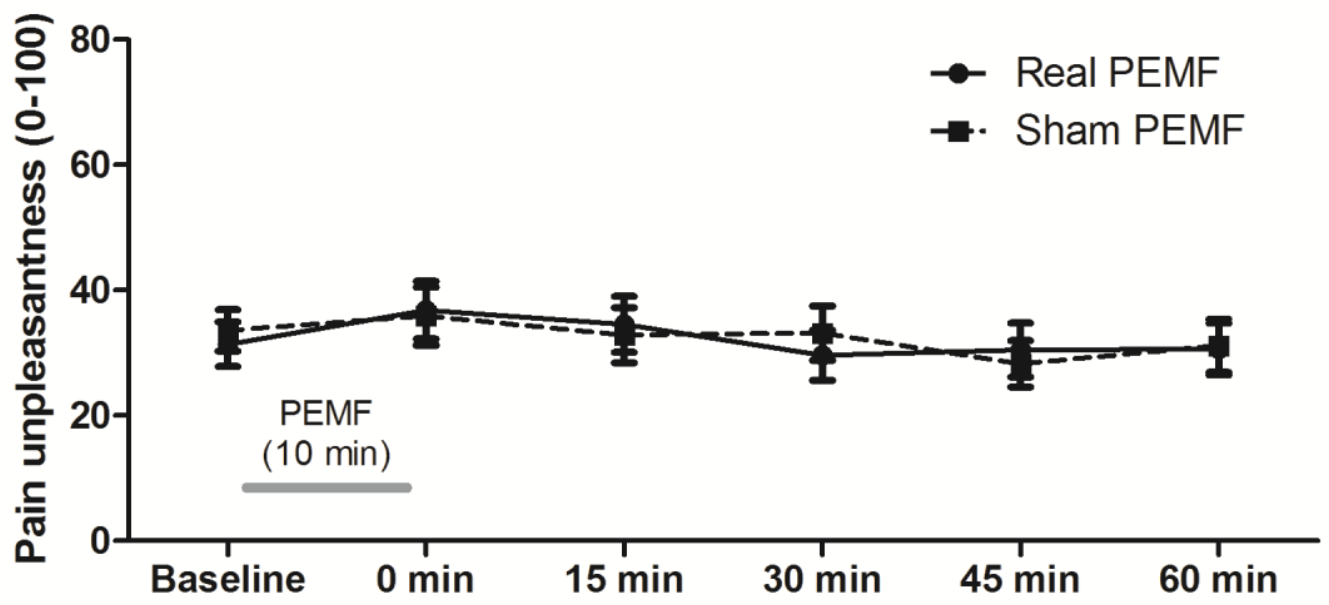




\section{Figure 2. Temporal summation}

Pain perception (pain intensity) gradually increased during the application of the tonic nociceptive stimuli. This gradual rise in pain was observed both before and immediately after real PEMF application.

$\rightarrow$ Baseline

-n. Post real PEMF

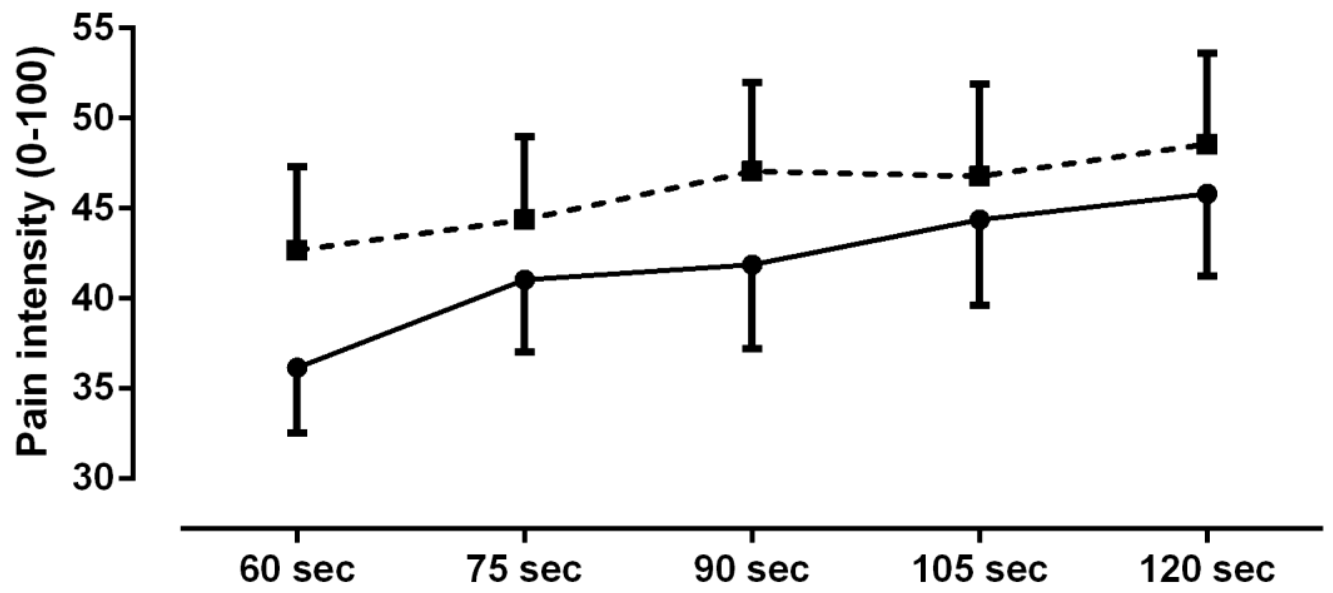

\section{Figure 3. Effect of PEMF on temporal summation}

The pain intensity scores were higher at $120 \mathrm{sec}$ compared to $60 \mathrm{sec}$, a phenomenon attributable to temporal summation. There was no difference between the temporal summation delta scores obtained at baseline, $0 \mathrm{~min}, 15 \mathrm{~min}, 30 \mathrm{~min}, 45 \mathrm{~min}$ and $60 \mathrm{~min}$ post-PEFM $(\mathrm{p}=.74)$.

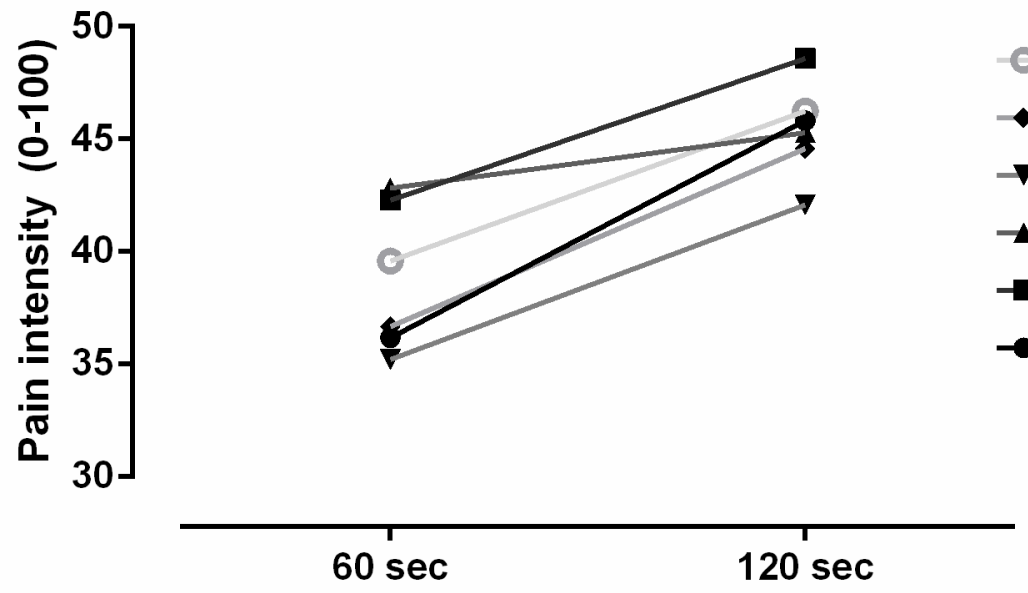

